\title{
Highlights from the ANTARES neutrino telescope
}

\author{
Alexander Enzenhöfer ${ }^{* \dagger}$ \\ CPPM, Marseilles, France \\ E-mail: enzenhoefer@cppm.in2p3.fr \\ on behalf of the ANTARES Collaboration
}

The ANTARES experiment has been running in its final configuration since 2008. It is the largest neutrino telescope in the Northern hemisphere. After the discovery of a cosmic neutrino diffuse flux by the IceCube detector, the search for its origin has become a key mission in high-energy astrophysics to which ANTARES can bring a valuable contribution.

The ANTARES sensitivity is large enough to constrain the origin of the IceCube excess from regions extended up to $0.2 \mathrm{sr}$ in the Southern sky. Assuming different spectral indexes for the energy spectrum of neutrino emitters, the Southern sky and in particular central regions of our Galaxy are studied searching for point-like objects and for extended regions of emission. The search program also includes multi-messenger analyses requiring time and/or space coincidences with other cosmic probes, for instance gamma rays as detected by Fermi. Recently, ANTARES has participated, with IceCube, to a high-energy neutrino follow-up of the gravitational wave signal GW150914, providing the first constraint on high energy neutrino emission from a binary black hole coalescence.

Finally, ANTARES has also provided performed indirect searches for Dark Matter: the limits obtained for the spin-dependent WIMP-nucleon cross section overcome that of existing directdetection experiments.

38th International Conference on High Energy Physics

3-10 August 2016

Chicago, USA

\footnotetext{
* Speaker.

${ }^{\dagger}$ On behalf of the ANTARES Collaboration.
} 


\section{Introduction}

The ANTARES detector [1] is a neutrino telescope located in the Mediterranean Sea off the coast of Toulon, France. It is anchored to the seabed at a depth of about $2500 \mathrm{~m}$. With its instrumented volume of approximately $0.01 \mathrm{~km}^{3}$, it is the largest neutrino telescope in the Northern Hemispehere. This instrumented volume comprises an array of $88510^{\prime \prime}$ photomultiplier tubes distributed over 12 vertical detection lines. ANTARES is designed primarily to search for $\mathrm{E} \geq 100 \mathrm{GeV}$ muons resulting from the charged-current interactions of $v_{\mu}$ in the vicinity of the detector through the detection of the Cherenkov light induced by the passage in water of ultrarelativistic charged particles. The detected photons allow for an energy estimation of the secondary particle produced by a neutrino interaction. The arrival times of the photons produced by the secondary particle can be used to determine the track of this particle with an angular resolution of a fraction of a degree for $\mathrm{E}_{v}$. Running in its final configuration since 2008, ANTARES enables a variety of different physics analysis. Ranging from high energy neutrino astrophysics over dark matter searches to the study of neutrino properties and neutrino oscillations. The main background for signals ANTARES is looking for, consists of atmospheric neutrinos and muons. In order to discriminate cosmic neutrinos from this huge background, the neutrino searches are focused on either an excess of events in small restricted solid angle regions or an excess of events above a given energy by exploiting the difference in the expected energy spectra or by using external triggers from other experiments to define candidates in temporal and/or spatial correlation to those.

Highlights from a wide range of analyses using ANTARES data are reported here.

\section{Diffuse Flux searches}

The search for a diffuse neutrino flux is based on the search for an excess in the flux produced by unresolved sources. In this approach, the neutrino flux is integrated over the full visible sky. A selection of the most energetic events is done using the fact that the energy spectrum for cosmic signals is expected to be harder than that of the background. This analysis can be done using tracks (results shown here) and/or showers to benefit from complimentary information provided by both channels. The result for high energy neutrinos is shown in Fig. 1. This spectrum was obtained using ANTARES data of reconstructed muon tracks from 2007 - 2015 with a total lifetime of 2451 days. The data set contains 19 events above the energy cut whereas $13_{-4}^{+3}$ events are expected from simulations of the atmospheric background. About three events would have been produced by a cosmic flux as observed by IceCube. The resulting limits on an $\mathrm{E}^{-2}$ flux are given in Fig. 2.

The diffuse flux searches in ANTARES are also performed on reduced search windows, confined regions in the sky of particular interest e.g. the Galactic Ridge or the so-called Fermi Bubbles. Combined diffuse flux analysis with IceCube are also performed to improve the overall sensitivity.

\section{Point Source searches}

The very good angular resolution for neutino-induced muon events and the use of adequate analysis techniques allow for a strong background suppression and allow for a search of neutrino point sources. The search is restricted to events coming from below or only from a few degrees 


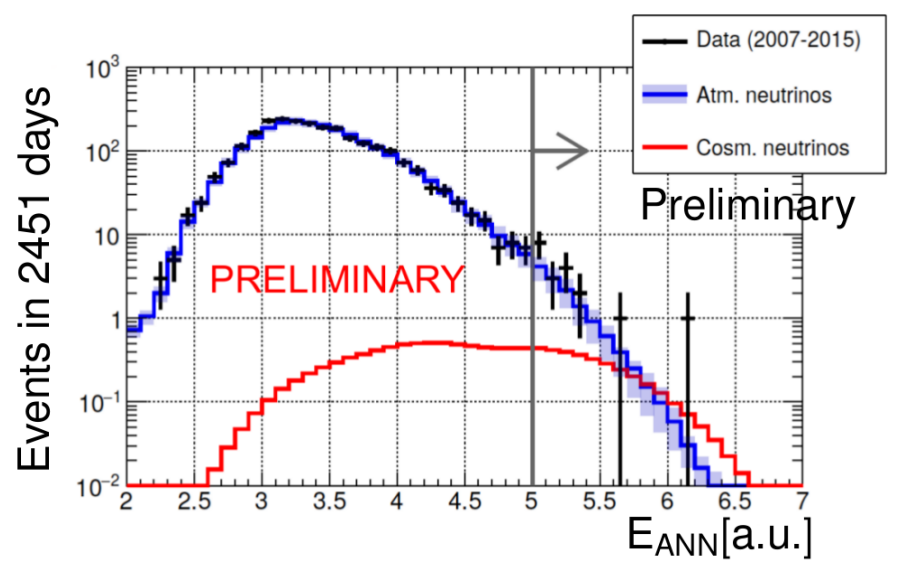

Figure 1: ANN energy distribution for data, in black, and Monte Carlo simulations, blue for atmospheric neutrinos and red for a cosmic signal analogous to the IceCube flux for $\mathrm{E}^{-2}$ spectrum.

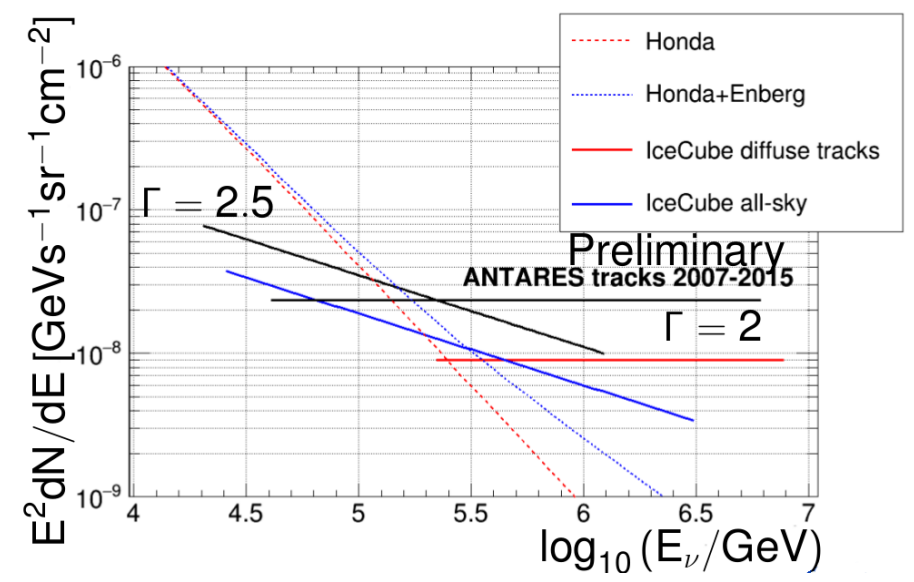

Figure 2: $90 \%$ C.L. upper limits for the track analysis, compared to the sensitivity achievable with the entire ANTARES 2007 - 2015 data sample.

above the horizon due to the high rate of downgoing muons. The remaining background thus mainly consists of the flux of atmospheric $v_{\mu}$ and mis-reconstructed atmospheric $\mu$. This yields a good sensitivity to neutrino sources located in the Southern Hemisphere. A combined search for pointlike sources including tracks and showers was performed using 1690 days of effective lifetime from 2007 - 2013. The data sample consisted of 6490 muon-track events and 172 shower events which passed the applied cuts. An estimated contamination of $10 \%$ mis-reconstructed atmospheric muons was assumed for both channels. The resulting skymap is shown in Fig. 4. An untargeted point-source search, a search over a list of pre-specified candidates, and a search using the origins of the IceCube events reported in Ref. [3] were applied to this data. No significant excess was observed. The resulting limits on point-like sources are given in Fig.3. While the atmospheric background produces predominantly muon-track events, an $\mathrm{E}^{2}$ point source with a flavour-uniform flux would be expected to produce a cascade-to-track ratio of 3:10, significantly increasing the 
sensitivity of the search. The sensitivity in this search was $\sim 10^{-8} \mathrm{GeV} \mathrm{cm}^{-2} \mathrm{~s}^{-1}$ for $\delta<-40^{\circ}$. A joint ANTARES and IceCube search for a neutrino excess from selected sources in the Southern hemisphere is detailed in Ref. [4].

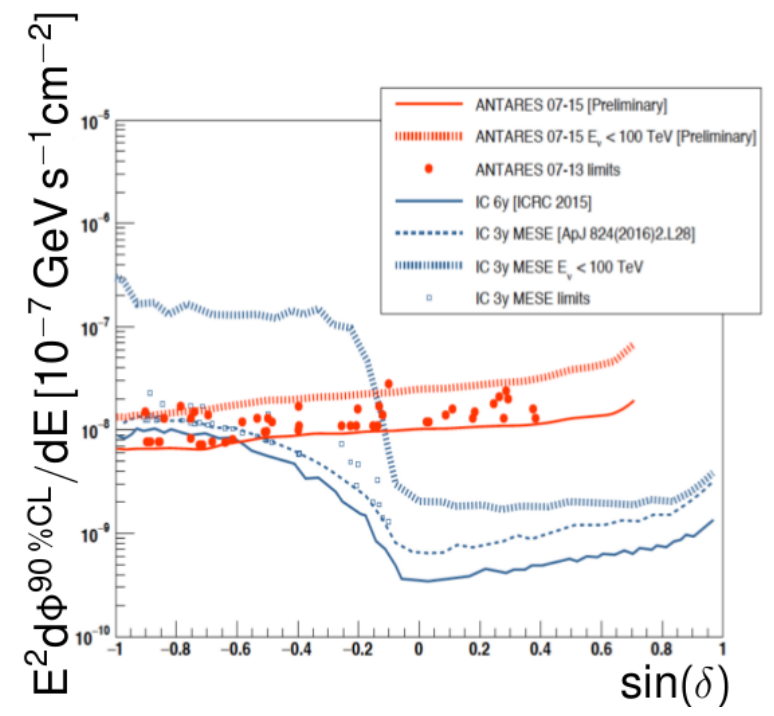

Figure 3: Sensitivities and limits for ANTARES (red) and IceCube (blue). Dots represent the limits. The sensitivities of ANTARES correspond to the all energy (continuous line) and $\mathrm{E}_{v}<100 \mathrm{TeV}$ (dashed line) sensitivities. The IceCube ones correspond to the 6y all-energy (continuous line), and $\mathrm{E}_{v}<100 \mathrm{TeV}$ (thick dashed line). For galactic sources, neutrinos below $100 \mathrm{TeV}$ are largely dominant.

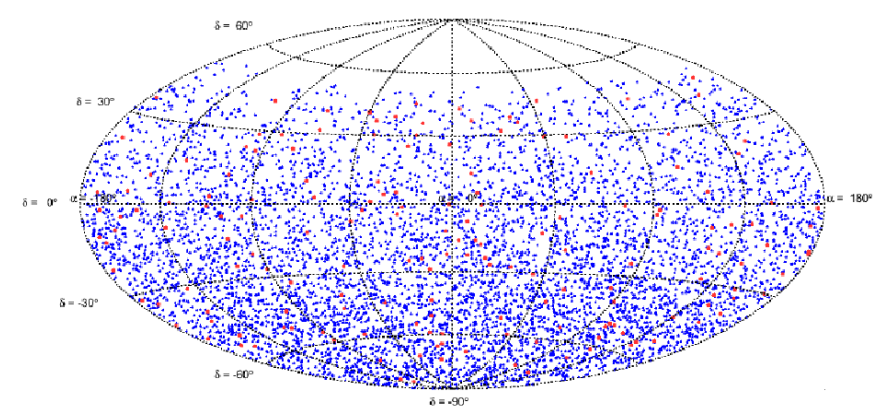

Figure 4: The arrival directions of events used in the ANTARES all-sky point-source-search sample. Track events are shown in blue, shower events in red.

\section{Multi Messenger Program}

ANTARES also maintains a multi messenger program to share data with other collaborations. This TAToO (Telescopes-ANTARES Target-of-Opportunity) program sends alert messages to associated instruments in order to trigger follow-up observations if an event passes certain quality criteria. Depending on the energy of the reconstructed particle, different telescopes may be alerted. 
Associated instruments include optical telescopes (TAROT, MASTER, ZADKO) [5], radio telesopes (MWA) [6], x-ray detectors (Swift-XRT) as well as ground-based $\gamma$-ray detectors (H.E.S.S, HAWC). The combined detection of different messengers from common sources can provide invaluable insight into the underlying physical processes. Limiting the searches in space and time leads to low background contributions and improve the instruments' sensitivity. In addition, uncorrelated backgrounds and systematics for different messengers help to further improve the results. ANTARES also undertakes other multi messenger efforts, outside the TAToO program, like the combined follow up of the GW150914 gravitational wave event with IceCube [7]. The result is presented in Fig. 5.

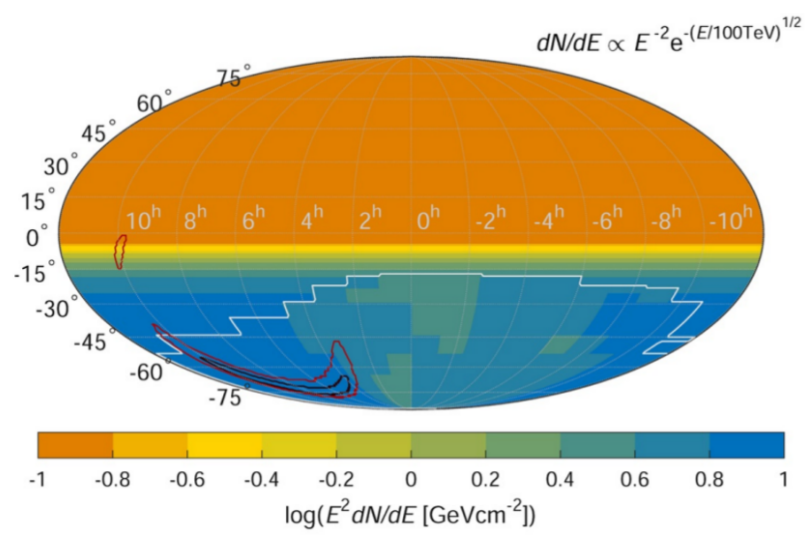

Figure 5: Upper limit on the high-energy $v_{\mu}+v_{\mu}$ spectral fluence from GW150914 as a function of source direction. The region surrounded by a white line shows the part of the sky in which ANTARES is more sensitive (close to nadir), while on the rest of the sky, IceCube is more sensitive. For comparison, the 50\% CL and $90 \%$ CL contours of the GW sky map are also shown (red lines on the bottom left).

\section{Dark Matter searches}

ANTARES can also place limits on different WIMP dark-matter scenarios by limiting the neutrino flux expected from WIMP interactions in the Sun, Earth, Galactic Centre, dwarf galaxies and galaxy clusters. Since the expected dark-matter density tends to be strongly peaked near the centres of these objects, and ANTARES has an excellent angular resolution, competitive limits can be set in the $\mathrm{E}_{\text {WIMP }} \gtrsim 50 \mathrm{GeV}$ range where ANTARES is sensitive. The geographical location of the detector is also an advantage compared to IceCube, since it allows a better visibility of the Galactic Center, being in the Northern hemisphere, and an observation of the Sun with less atmospheric background, being at intermediate latitude (and therefore observing the Sun less close to the horizon). A search for a neutrino flux from the direction of the Galactic Center with data from $2007-2015$ was used to set limits on the WIMP-WIMP velocity-averaged self-annihilation cross section $\left\langle\sigma_{\mathrm{A}} \mathrm{v}\right\rangle$ shown in Fig. 6. These limits are the most competitive ones for neutrino telescopes given this better visibility of the Galactic Centre compared to the South Pole [8]. Neutrino telescopes produce the more stringent limits in the $\mathrm{M}_{\mathrm{WIMP}}>200 \mathrm{GeV}$ range, surpassing even the 
direct-detection experiments. Further dark-matter analyses by ANTARES includes a search for a WIMP signature from the Sun [9] and a test of secluded dark-matter models in the Sun [10].

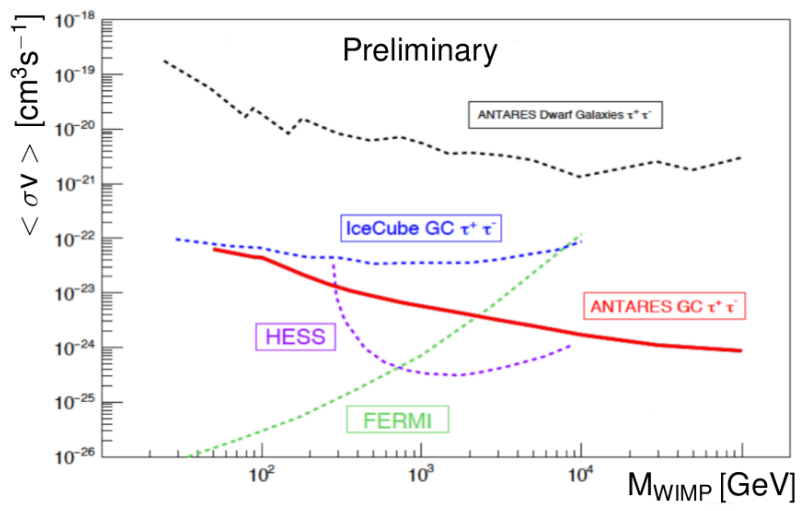

Figure 6: ANTARES limits on $\left\langle\sigma_{\mathrm{A}} \mathrm{v}\right\rangle$ from the Galactic Centre as a function of the WIMP mass.

\section{Conclusion}

ANTARES already demonstrated the great potential of deep sea neutrino observatories and will continue to improve its performance during its final stage of operation. Although limited by its small instrumented volume, analysis performed on ANTARES data delivers competitive results. These results together with the detection of cosmic neutrinos by IceCube [2][3] changed the perception of neutrino astronomy and paved the way for a future multi-km ${ }^{3}$ detector in the Mediterranean Sea [11]. This next generation neutrino telescope is currently under construction as KM3NeT Phase 1 and will have an instrumented volume of about three times larger than that of ANTARES. As KM3NeT grows larger, its sensitivity will improve and eventually reaches the necessary level for discoveries.

\section{References}

[1] M. Ageron et al., Nuclear Instruments and Methods in Physics Research A 656 (2011) 11.

[2] M. G. Aartsen et al., Science 342 (2013) 1242856.

[3] M. G. Aartsen et al., PRL 113 (2014) 101101.

[4] S. Adrián-Martínez et al., ApJ. 823 (2016) 65.

[5] S. Adrián-Martínez et al., JCAP 1602 (2016) 02, 062.

[6] S. Croft et al., Astrophys.J. 820 (2016) 2, L24.

[7] S. Adrián-Martínez et al., PRD 93 (2016) 122010.

[8] M. G. Aartsen et al., arXiv:1606.00209.

[9] S. Adrián-Martínez et al., Physics Letters B, 759 (2016) 69-74.

[10] S. Adrián-Martínez et al., JCAP 05 (2016) 016.

[11] S. Adrián-Martínez et al., J.Phys. G 43 (2016) 8, 084001. 